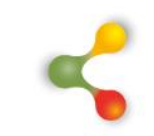

REVUE HYBRIDE DE L'ÉDUCATION

Conférence d'ouverture du colloque Apprendre du, au et par le travail en formation professionnelle

\title{
S'écarter du travail pour mieux apprendre le travail
}

Patrick Mayen, professeur en sciences de l'éducation à l'Université de Bourgogne Franche-Comté/Agrosup Dijon. Unité de Recherche Développement professionnel et formation, France,

patrick.mayen@educagri.fr 


\section{REVUE HYBRIDE DE L'ÉDUCATION}

\section{Note}

Lors du $86^{\mathrm{e}}$ congrès de l'ACFAS en mai 2018 - plus particulièrement dans le cadre du colloque 517 de la section Éducation, intitulé Apprendre du, au et par le travail en formation professionnelle - , nous avons fait la rencontre du chercheur Patrick Mayen qui a prononcé la conférence d'ouverture S'écarter du travail pour mieux apprendre le travail. Ce chercheur nous invite à conjuguer avec style les verbes AGIR et APPRENDRE. Ainsi, la didactique professionnelle propose-t-elle diverses manières de transformer les actions en situation de travail afin que celles-ci soient plus faciles à apprendre.

Quels éléments de l'environnement de travail peut-on inclure, exclure ou moduler afin de concevoir de meilleurs environnements d'apprentissage en situation de travail? Comment évaluer et maximiser le potentiel d'apprentissage de différentes situations de travail ? Comment soutenir le développement des processus décisionnels et des approches participatives et réflexives en milieu de travail ? Comment faciliter le partage des savoirs expérientiels à l'intérieur d'une communauté apprenante au travail?

La travailleuse ou le travailleur apprenant, qu'elle ou il produise des biens ou des services, exerce un raisonnement, mobilise des savoirs de plus en plus complexes, pose des diagnostics et évalue les effets de ses actions. Quelles que soient les configurations et les organisations du milieu de travail, celui-ci doit promouvoir le développement de l'agir professionnel. 


\section{$\&$}

\section{REVUE HYBRIDE DE L'ÉDUCATION}

\section{Introduction}

Je voudrais orienter cet article vers une réflexion fondée sur une série de recherches et de constats; réflexion selon laquelle, pour apprendre et se former dans et par le travail, il est souvent nécessaire de s'écarter du travail. En cohérence avec cette orientation, il semblerait utile que ce qu'on peut appeler l'ingénierie des formations en situation de travail, et plus largement, celle de la conception d'organisations ou d'environnements apprenants, intègre aussi cette réflexion.

Dans les lignes qui suivent, je développerai tout d'abord les origines de cette réflexion, puis dans une visée d'opérationnalité, je proposerai de décrire cinq formes possibles de formation en milieu de travail, toutes visant à ce que des personnes fassent des expériences apprenantes, à la fois auprès du travail, mais toujours à distance de celui-ci.

J'emprunte l'idée d'écart, de décalage, de détour, de décoïncidence au philosophe et sinologue François Jullien dont l'œuvre philosophique a consisté à penser la philosophie occidentale à partir d'un détour par la pensée chinoise (Jullien, 2017). Comme il l'écrit à ce propos : il s'agit d'en sortir pour mieux y revenir. En le paraphrasant, je dirais qu'il est souvent nécessaire, pour apprendre le travail, de s'écarter du travail. Pour prendre le vocabulaire des ergonomes, je pourrais dire qu'il peut être utile de s'écarter du travail réel, pour mieux apprendre à maîtriser le travail réel.

\section{Styliser, au cœur du travail}

Le psychologue Jérôme Bruner, dont les travaux sur la médiation et le tutorat ont exercé une grande influence, utilise une notion étonnante pour décrire l'activité d'un grand nombre de personnes qui sont amenées à exercer une fonction de tutelle, encore désignée par le terme d'étayage auprès de personnes moins expérimentées ou moins compétentes (1983, 1988, 2000). Bruner observe, dans des contextes différents, que les adultes «stylisent » leur action pour la mettre en scène, pour la montrer, pour la soumettre à l'imitation. Mais que veut dire "styliser »? Styliser signifie transformer l'action telle que le tuteur la réaliserait s'il était seul, pour la mettre à portée de celui qui apprend. Styliser ne concerne pas que les apprentissages entre adultes et enfants; cela concerne aussi les apprentissages professionnels entre plus expérimentés et nouveaux. Lorsqu'un tuteur stylise son action, il peut utiliser différents procédés : ralentir, outrer son geste, ralentir voire arrêter l'action à des moments critiques, recommencer, s'aider de la parole pour faire ressortir les caractéristiques essentielles vers lesquelles l'apprenant doit diriger son attention, accroître la complexité de l'action ou de l'interaction avec celui qui apprend. II peut aussi mettre en scène l'environnement de la tâche de manière à ce que l'environnement soit simplifié ou débarrassé d'éléments parasites. J'avais pu observer, dans une recherche auprès de tuteurs 


\section{$\&$}

\section{REVUE HYBRIDE DE L'ÉDUCATION}

facteurs, que certains d'entre eux opéraient effectivement de la sorte (Mayen, 2014).

Préoccupés de faire apprendre aux futurs facteurs des pratiques qui ne les mettraient pas en danger, ils préparaient le terrain, y compris avec certains clients. En présence de leur apprenti, ils ne réalisaient pas tout à fait la même tournée et ne travaillaient pas tout à fait comme d'habitude. Ils se tenaient plus près de la réglementation, d'une part, et ils effectuaient les actions de manière plus «tenue » d'autre part. Cela ne signifie pas qu'ils travaillaient approximativement en l'absence d'un apprenti. Seuls, ils se reposaient davantage sur leur expérience fine de leur tournée, empruntaient des raccourcis pour réaliser l'action, s'autorisaient des variations. Ainsi, leurs propos étant explicites à cet égard, en présence d'un apprenti, ils montraient et disaient le travail pour un apprenti.

Ces stratégies, marquées par leur attention pour les futurs facteurs, ne sont pas nécessairement partagées par tous les professionnels qui exercent à un moment ou un autre une fonction de tutorat. Ce que nous en retenons ici, c'est l'intentionnalité de l'écart ou du détour par la mise en scène d'un environnement redéfini pour l'apprentissage.

La notion de stylisation me paraît représentative de ces opérations de décalage, de décoïncidence (Jullien, 2017) et d'écart, qui consistent à s'écarter de la tâche et de l'action telles qu'elles se déploient dans le monde de l'action, pour faire un détour qui vise l'apprentissage. On pourrait parler de mise en scène pédagogique ou didactique, de didactisation ou de ruse pédagogique ou didactique.

Concevoir ou aménager les conditions d'apprentissage au travail consiste aussi, dans de nombreux cas, et selon différentes modalités, à styliser le travail pour le rendre apprenant.

\section{Apprendre des situations}

Dans un numéro de la revue Éducation permanente intitulé Apprendre des situations, Pierre Pastré (1999) soulignait le double sens de ce titre: un des objectifs de la formation professionnelle consiste à apprendre les situations au sens « d'apprendre à les maîtriser », d'une part, et d'autre part, consiste à apprendre des situations au sens "d'apprendre d'elles » ou "d'apprendre de l'activité avec les situations ». La notion de situation est centrale puisqu'elle occupe ici deux places : la situation but, à maîtriser, et la situation comme moyen, en l'occurrence, moyen pour apprendre.

En didactique professionnelle, les situations de travail ont aussi une autre place : celle d'origine. La didactique professionnelle est définie depuis sa création comme l'analyse du travail pour la formation, avec l'idée selon laquelle il est très utile d'analyser les situations de travail pour concevoir les formations : 


\section{$\&$}

\section{REVUE HYBRIDE DE L'ÉDUCATION}

- Pour identifier les capacités et connaissances requises pour l'action efficiente en situation ;

- Pour identifier le potentiel d'apprentissage des situations de travail (Mayen, 2018 ; Mayen et Gagneur, 2017).

Apprendre de et par les situations de travail ou former par l'activité et l'expérience avec les situations de travail, suppose donc d'identifier au préalable le potentiel d'apprentissage des situations, soit pour les utiliser en tant que situations formatives par elles-mêmes, soit pour les aménager afin d'accroître leur potentiel d'apprentissage, soit pour intervenir sur l'activité de celui qui apprend pour accroître le potentiel d'apprentissage de l'activité avec ces situations.

C'est là que se situe l'objet principal de cet article. Dans un très grand nombre de cas, les situations de travail ne sont pas ou peu apprenantes ; elles peuvent se révéler dés-apprenantes ou conduire à des apprentissages négatifs ou inutiles. En outre, l'expérience de ces situations et avec ces situations peut s'avérer peu efficiente ; l'apprentissage sur le tas peut être plus long, plus difficile, limiter ou empêcher certains apprentissages. Enfin, la direction des apprentissages est incertaine. On peut apprendre par l'activité en situation de travail, mais quoi ?

En ce sens, pour utiliser à son maximum le potentiel apprenant ou formateur du travail au sens d'activité avec des situations, il est nécessaire de s'en écarter, de faire décoïncider "activité tournée vers des objectifs productifs » et « activité tournée vers des objectifs d'apprentissage », de les décaler.

\section{La notion de potentiel d'apprentissage}

La notion de potentiel d'apprentissage des situations cherche à rendre compte des possibilités d'apprentissage qu'une situation ou un ensemble de situations de travail peut offrir à l'activité d'un individu à un certain moment de sa vie. Même si une situation, vue comme un environnement donné, comporte des conditions a priori plus ou moins favorables pour engager et soutenir des processus d'apprentissages, de manière générale, il est toujours nécessaire de rapporter ce potentiel général à l'état des capacités de chaque individu. Plus exactement, si l'on prend au sérieux la notion de zone de proche développement de Vygotski, il est nécessaire de rapporter le potentiel d'apprentissage d'une situation avec non seulement ce qu'une personne sait ou sait déjà faire, mais aussi avec ce qu'elle est capable de faire avec un environnement aidant.

La notion de potentiel d'apprentissage d'une situation ou d'un ensemble de situations part des constats selon lesquels les milieux de travail et les situations qui les composent et les expériences que des individus vivent dans et avec ces situations peuvent avoir un potentiel 


\section{6}

\section{REVUE HYBRIDE DE L'ÉDUCATION}

d'apprentissage qui va du plus élevé au plus bas, voire au plus négatif. Ainsi, l'expérience du travail dans un certain milieu peut se révéler très positive et engendrer des apprentissages et du développement de niveau élevé ou peut, à l'opposé, se révéler neutre. On ne désapprend pas, mais on n'apprend rien de plus. Elle peut aussi se révéler négative. Dans ce cas, les connaissances et les capacités peuvent se dégrader, se scléroser, devenir obsolètes, etc.

La notion de potentiel d'apprentissage correspond au potentiel proposé par les conditions qui composent une situation ou un ensemble de situations et par la nature des activités qu'elle suscite chez les individus qui agissent dans, avec, et sur cette situation.

Prenons un exemple que j'aime développer : dans certains garages d'entretien et de réparation automobile, on peut observer que les techniciens ont la possibilité de faire appel à un collègue en cas de panne complexe pour les aider à effectuer un diagnostic ou encore, en cas d'opérations délicates ou complexes. Ils peuvent aussi appeler leurs collègues lorsqu'ils sont en présence d'un nouveau système technique ou d'un nouveau modèle de voiture afin de partager leur découverte. Dans ces entreprises, lorsqu'un nouveau modèle ou un nouveau système apparaît, des moments de formation sont organisés. C'est donc l'occasion d'entretenir et d'actualiser ses connaissances. À l'inverse, une observation récente de Sarah Macler dans sa thèse sur les parcours et les compétences de chefs de très petites entreprises montre un petit garage dans lequel le chef d'entreprise, pourtant préoccupé des compétences de ses deux employés, prend systématiquement en charge les interventions les plus complexes qui arrivent dans son garage. II n'a pas conscience qu'il prive ainsi ses techniciens de l'occasion de mobiliser leurs capacités et connaissances les plus pointues, d'entraîner ces capacités, de les développer et de les actualiser par la confrontation à des situations problèmes exigeantes. Le milieu de travail qu'il crée ainsi, sans en avoir conscience, est peu apprenant voire désapprenant.

\section{Quatre formes d'apprentissage par l'expérience des situations de travail}

On peut considérer quatre formes de configurations d'apprentissage par l'expérience des situations de travail. La première forme a déjà été évoquée, il s'agit de l'apprentissage sur le tas. A priori, c'est elle qui fait le moins l'objet de l'activité des formateurs ou de ceux qui veulent faire du travail un espace et un moyen de construction et de développement des connaissances et des capacités. Les trois autres formes supposent ce qu'on appelle en France des activités d'ingénierie des compétences pour prendre le terme de Le Boterf, ou d'ingénierie de formation. Ce sont ces trois formes qui nous intéressent ici, car elles demandent à la fois une proximité avec le travail (les situations et l'activité avec les situations) et 


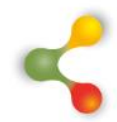

\section{REVUE HYBRIDE DE L'ÉDUCATION}

une prise de distance, un découplage, une décoïncidence, un écart avec le travail, ou tel qu'il est nommé par les ergonomes, avec le travail « réel ».

La deuxième forme est donc l'aménagement des environnements, autrement dit, des conditions physiques, techniques et organisationnelles du travail pour le rendre plus apprenant ou formateur. On parle souvent ici d'organisation ou d'entreprise apprenante.

La troisième forme correspond à l'action intentionnelle d'aide ou de formation en situation de travail. Par la présence d'un ou de plusieurs autres, la situation est transformée et une médiation introduit une transformation des interactions entre celui qui apprend et la situation en tant qu'environnement donné.

La quatrième forme peut être désignée par les termes de circulation ou encore de parcours dans le milieu de travail, au-delà et en dehors du périmètre de l'emploi, du poste, des tâches ou d'un individu. II s'agit alors de faire explorer ou " expériencier " par celui qui apprend, des situations qui font partie de son environnement global, mais qu'il ne connaît pas ou peu. Par exemple, explorer l'amont et l'aval du poste de travail pour comprendre les tenants et aboutissants de son action ou explorer la position du client, tout cela afin d'élargir et d'enrichir le champ de sa représentation.

\section{Analyser les situations au regard de leurs exigences d'apprentissage et de leur potentiel d'apprentissage : l'analyse didactique professionnelle du travail}

L'un des outils principaux de l'analyse du travail pour la formation, au-delà de la conception et de la conduite des formations, réfère à nos connaissances dans les processus d'apprentissage. II paraît évident que lorsque nous décidons de proposer à des apprenants certaines combinaisons d'activités, c'est parce que nous savons que susciter et étayer ce type d'activités constitue un facteur d'apprentissage ou plus exactement, une combinaison favorable à des apprentissages. Nous favorisons par exemple, des activités de déstabilisation ou de contradiction, des activités d'entraînement ou de répétition, nous intervenons pour diriger l'attention vers un aspect d'un problème ignoré par l'apprenant, etc. De la même manière, nous pouvons utiliser nos connaissances concernant les conditions et les processus d'apprentissage pour l'analyse du travail, des conditions du travail et des formes d'activité qu'il suscite et soutient.

Ainsi, dans une recherche conduite à propos du travail et de la formation dans le domaine des travaux paysagers, nous avons pu observer que selon le mode d'encadrement des équipes, chacun des ouvriers pouvait ou non avoir accès aux opérations d'orientation de l'action. Dans certaines équipes, seul le chef d'équipe effectue les activités de diagnostic du chantier, décide des opérations à réaliser, exerce des activités 


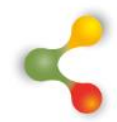

\section{REVUE HYBRIDE DE L'ÉDUCATION}

continues de contrôle de l'avancée du chantier. II prive ainsi les ouvriers et les apprentis de ces activités les plus exigeantes cognitivement. Pour les anciens, ils risquent de perdre une part de leurs compétences puisqu'ils ne les exercent plus. Pour les apprentis, ils n'ont accès qu'à une part restreinte des activités du métier, ce qui limite leurs apprentissages à l'exécution d'actions décidées par un autre.

Une intervention en ingénierie des compétences consiste alors à intervenir sur les conditions du travail - ici des conditions organisationnelles et managériales - afin de faire en sorte que, comme dans d'autres équipes, le chef analyse le chantier et définisse le travail à faire avec ses ouvriers, sollicitant et reconnaissant leur activité de diagnostic, leurs propositions, etc. II peut organiser le travail afin que chacun ait la responsabilité d'un segment plus large du chantier, organiser des rotations afin de ne pas spécialiser ses ouvriers, mais au contraire, entraîner et élargir leur champ de compétence.

Le philosophe John Dewey distingue "avoir une expérience » et " faire une expérience ». Une expérience eue ne sollicite pas ce que Dewey appelle le schème de l'enquête. Autrement dit, elle ne sollicite pas la mise en mouvement de l'intelligence, de l'attention, de l'analyse raisonnée de la situation, de l'imagination, de la construction d'hypothèses et de la décision d'action dont les conséquences sont évaluées.

\section{Forme 1 : Apprendre sur le tas}

Si nous prenons le temps ici de définir quelques caractéristiques de ce qu'est l'apprentissage sur le tas, c'est dans le but de marquer les différences avec les autres formes que nous allons développer.

- Il est dirigé par le cours du travail, c'est-à-dire que ce qui s'apprend dépend de ce qui arrive dans la situation, des tâches et événements qui se présentent. C'est l'action qui est première et l'apprentissage résulte des nécessités de l'action.

- Dans le même sens, l'apprentissage sur le tas est fortement lié aux situations spécifiques dans lesquelles il s'effectue. On dit aussi qu'il s'agit d'un apprentissage local, hautement contextualisé. Ici se pose la question de la généralisation ou de la transférabilité de ce qui est appris face à des situations de la même famille, mais qui présentent des caractéristiques propres?

- Il est plutôt et plus souvent implicite, bien que, en tous les cas, peu explicite à celui-là même qui apprend.

- II n'est pas systématique, mais opportuniste. II se réalise jusque-là où le cours de l'action peut s'engager ou reprendre quand il est interrompu. Autrement dit, il est intrinsèquement lié à la réussite de l'action. II s'agit de parvenir à agir et non pas de comprendre et d'apprendre. 


\section{6}

\section{REVUE HYBRIDE DE L'ÉDUCATION}

- Les ressources sont locales et immédiates. Autrement dit, celui qui apprend recours aux aides, documents, sources d'information disponibles et pratiques. De même, il est dépendant de l'entourage pour imiter, pour questionner, pour recevoir de l'aide ou des explications.

Une question spécifique se pose qui est celle de l'intentionnalité d'apprendre. C'est un facteur essentiel dans tout apprentissage. II correspond à une question sur l'activité effective de chaque individu dans ces situations. II peut être dirigé par le faire et la réussite, et les séquences de recherche d'information, d'attention dirigée vers les effets de son action, d'imitation d'un collègue expérimenté, etc., n'ont pour but que de pouvoir ajuster son action afin de répondre aux exigences de la tâche. L'intention d'apprendre n'est pas présente à la conscience : d'être à la fois en situation d'action et en situation d'apprentissage, de faire et de chercher à apprendre modifie l'activité en situation. On observe ainsi chez des apprentis une recherche délibérée d'apprentissage et de développement : par exemple, des apprentis font et refont ou recherchent des modèles de résultats (produits finis), comparent leur production avec des modèles de référence, posent davantage de questions à l'entourage ou sollicitent de la validation ou des critiques sur ce qu'ils ont produit ou sur leur manière de s'y prendre. Certains prennent des notes pour conserver des repères d'action ou noter une procédure réussie ou des points d'attention ou de risques. Enfin, l'intention d'apprendre peut aussi se manifester par une activité de contrôle des activités pour apprendre, du type "se dire qu'il faudra faire attention à tel événement la prochaine fois » ou encore, se redire la procédure réussie pour la mémoriser, etc.

II faudrait donc, mais ce n'est pas le but de cette conférence, distinguer apprentissage sur le tas implicite et non intentionnel, et apprentissage sur le tas avec intention ; l'intention, consciente et volontaire, comporte déjà une forme de ce que nous avons appelé : distance, décalage, découplage, écart au travail. C'est pourquoi l'apprentissage sur le tas, mais dans une visée intentionnelle d'apprentissage, est déjà une forme d'apprentissage distanciée de l'apprentissage sur le tas, du travail tel qu'il est à faire et tel qu'il se réalise.

\section{Forme 2 : L'environnement apprenant}

L'exemple des garages et du potentiel d'apprentissage de l'environnement technique, organisationnel, managérial, etc. a été développé ici. La question principale est toujours la même : quelle activité suscite la nature du travail et des situations avec lesquels une personne a affaire ? Dans quelle mesure le travail présente-t-il des variations propres à exiger la construction et le développement de capacités d'ajustement aux variations ? Est-il, au contraire, tellement répétitif qu'il sollicite le même schème d'action? Dans quelle mesure le travail conduit-il à faire 


\section{6}

\section{REVUE HYBRIDE DE L'ÉDUCATION}

l'expérience de la diversité des situations professionnelles de la même famille? Par exemple, pour un professionnel qui exerce des fonctions de service aux personnes, a-t-il l'occasion de travailler avec des personnes d'âges différents, de pathologies différentes, dans des espaces de travail différents? Dans quelle mesure le travail permet-il ou non d'essayer et d'expérimenter ? Celui qui travaille peut-il accéder aux effets de son action afin de réguler celle-ci et d'apprendre par mise en relation entre les actions, conditions de l'action et effets de celle-ci? Selon les réponses à ces questions, le potentiel d'apprentissage peut être estimé plus ou moins élevé. II faut souligner que, pour les professionnels en place, la question de l'apprentissage se pose en termes de maintenance de ses capacités : par exemple, quelle est la part des capacités d'une personne qui est mobilisée par les tâches qui lui sont confiées? Quelle est sa marge d'autonomie? Quelle part des diagnostics et des décisions peut-elle prendre?

L'action du formateur peut intervenir sur les environnements en cherchant à les rendre plus apprenants. Cet aménagement du milieu peut aller d'intervention sur le contenu du travail demandé en modifiant le périmètre de l'emploi d'une personne, en modifiant la part d'intelligence de l'action qui lui est assignée, en l'associant pour participer à des diagnostics communs ou à des prises de décisions collectives ou encore, en lui donnant les possibilités d'accéder aux effets de son action, etc. On note ici que l'aménagement de l'environnement dans cette acception conserve ses caractéristiques productives, autrement dit, il reste organisé pour produire, même s'il constitue des conditions pour que des personnes apprennent par leur activité de travail.

L'aménagement peut aller plus loin et devenir formatif puisque celui qui apprend peut bénéficier de moments où les exigences de production sont suspendues ou allégées, par exemple, pour démonter et comprendre le système technique avec lequel il agit, pour s'entraîner et refaire un dossier, une action, une intervention, pour accéder à de l'information, pour comprendre, apprendre ou faire autrement. Dans certains lieux de travail, on peut réserver des machines, des pièces, des appareils qui servent d'entraînement.

\section{Forme 3 : la circulation dans le milieu ou l'organisation de parcours}

Cette forme est assez traditionnellement proposée aux cadres ou futurs cadres et ingénieurs. Elle consiste à "sortir » de l'espace de son périmètre de travail pour découvrir et explorer des espaces environnants. La circulation hors de son travail comporte un potentiel d'apprentissage important. De quelle manière ? Ici, deux processus d'apprentissage sont à l'œuvre : le changement de point de vue, d'une part, et l'élargissement du champ de sa représentation, d'autre part. 


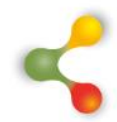

\section{REVUE HYBRIDE DE L'ÉDUCATION}

Les organisations ont tendance à délimiter un périmètre de travail, constitué des tâches à effectuer. Cette tendance s'accompagne d'un implicite selon lequel il ne serait pas utile de connaître ce qui intervient avant, après, parallèlement, conjointement à ce qui se passe dans le périmètre de l'emploi ou du poste. Pourtant, le fait que tout travail soit inséré dans un processus plus large conduit à ce qu'une situation, une tâche, soit en interrelation avec les situations et les tâches qui interviennent dans le processus plus global. Sur le plan cognitif, cependant, on peut observer que le périmètre de ce qui doit être connu et compris peut excéder le périmètre de l'action. On a là une première variante du découplage. John Dewey écrit que l'une des formes de croissance peut être identifiée, lorsqu'une part de l'environnement entre dans la situation. Autrement dit, lorsque, pour une personne, une partie de ce qui n'était que contexte ou de ce qui restait ignoré entre dans le champ de ce qui est agissant, qu'il peut prendre en compte, sur quoi il peut éventuellement agir.

Dans une formation adressée à des réceptionnistes de concessionnaires automobiles qui reçoivent des clients pour un entretien ou une réparation, j'avais ainsi utilisé une frise élaborée par un constructeur automobile qui décrivait tout ce qu'un client ressent, pense et fait, lorsque par obligation, il doit faire entretenir ou réparer sa voiture. La confrontation à cet aspect " externe » du travail avait été complétée par une enquête que les réceptionnistes pouvaient faire, dans un autre concessionnaire, en se mêlant aux clients qui attendent, en les observant et en observant leurs dialogues avec d'autres réceptionnistes, en leur posant un certain nombre de questions à propos de la réception.

Ces activités de circulation autour du travail et non dans leur propre travail avaient pour but d'étendre le champ de la représentation du processus d'entretien et de réparation automobile en l'étendant au ressenti et aux réflexions et aux actions des clients. II s'agissait aussi de modifier les interprétations des comportements des clients, stéréotypées et négatives, et celui des réceptionnistes également égocentrées.

Elles amenaient à une activité de changement de point de vue et d'élargissement du champ de la représentation, de construction de relations de cause à effet, aussi bien du pourquoi des comportements des clients, que des conséquences sur le comportement des clients de l'organisation du service et de la qualité de celui-ci.

Une deuxième variation de la circulation dans son travail peut être identifiée que l'on peut appeler une forme réflexive. II s'agit de l'analyse de son propre travail. De nombreux travaux en ergonomie de la formation ont mis en évidence le rôle de formation de l'auto-analyse de son travail par des professionnels à l'aide d'outils conceptuels et de méthodes issues de l'ergonomie ou de la psychologie du travail : analyse systématique de la tâche prescrite, analyse des buts, des résultats et des conditions d'action, identification des stratégies et règles d'action des professionnels et 


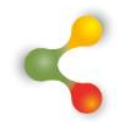

\section{REVUE HYBRIDE DE L'ÉDUCATION}

comparaison entre elles, analyse des justifications des règles et stratégies suivies, co-résolution de problèmes ou analyse collective de problèmes rencontrés. On observe là une série de modalités de prise de distance avec le travail. Dans un chantier en entreprise de l'industrie agroalimentaire, nous avions conduit une formation de cette sorte avec une cinquantaine d'ouvriers dans un atelier qui fonctionnait mal, et pour lequel les ouvriers n'avaient jamais formés. Nous avions fait le choix, après une analyse, d'organiser la formation en situation de travail en demandant à l'entreprise d'arrêter la production pendant les séquences de formation et de demander aux cadres et aux responsables des services de maintenance de se tenir à disposition des ouvriers qui allaient leur poser des questions et demander des explications sur leur système technique de production.

Dans un premier temps, chacun a été mis en situation de démonter sa machine ou le segment sur lequel il travaillait le plus souvent; le but étant de schématiser le fonctionnement du système pour pouvoir l'expliquer plus tard. II s'agissait aussi d'identifier ce qui fonctionnait plus ou moins bien et de proposer des améliorations. Dans un second temps, par paire, les ouvriers démontaient et s'expliquaient mutuellement le fonctionnement d'un segment plus large et devaient le schématiser. Progressivement, tout l'atelier devait être découvert, compris et expliqué par tous et un schéma normé commun devait représenter l'ensemble du processus en cours et une proposition de processus amélioré. Les ouvriers ont pu accéder aux documents techniques, aux informations informatisées, à certains savoirs des cadres ou de la maintenance. Les effets d'apprentissage ont été massifs ainsi que les propositions d'améliorations techniques et organisationnelles.

\section{Forme 4 : l'aide des autres}

Je ne ferai qu'évoquer ici cette forme de transformation des conditions d'apprentissage. Elle est la plus connue, même si elle n'est pas envisagée dans cette perspective d'aménagement et de décalage avec le travail lui-même. II est vrai que selon les formes de compagnonnage ou de tutorat, cette forme de transformation peut plus ou moins se rapprocher du travail, susciter des prises de distance, des détours et des configurations que la seule confrontation aux situations ne peut pas apporter, ni même offrir de l'aide simple, guidée par la réussite immédiate de l'action dans une stricte finalité de production.

Dans la théorie de Vygotski, la notion de zone proximale de développement est définie comme ce qu'une personne peut faire avec l'aide d'une autre. Cette première idée de la psychologie culturelle s'étend aux environnements ; ce qu'on peut énoncer ainsi, ce qu'une personne peut faire et apprendre dans un environnement au potentiel d'apprentissage élevé. Aménager un environnement revient ainsi à construire les étayages propres à stimuler et soutenir certaines activités qu'une personne ne ferait pas seule ou pas de la même manière. Dès lors 


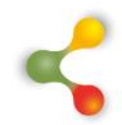

\section{REVUE HYBRIDE DE L'ÉDUCATION}

qu'une personne est présente dans l'environnement de celui qui apprend, elle influence son apprentissage, par simple imitation et mimétisme, par ses interventions pour aider, pour donner un conseil ou un coup de main, pour corriger un geste, pour expliquer un phénomène, etc. L'aide devient formellement formative lorsqu'une personne de l'entourage se voit confier un rôle de tutorat, de compagnonnage, de coaching ou de formation. $\mathrm{Ce}$ sont alors toutes les activités de cette nature qui peuvent être mobilisées. Elles sont cependant de deux sortes.

La première fonction consiste à aménager et organiser les situations pour les rendre plus formatives et servir les objectifs d'apprentissage. Ce que n'en fait pas une aide occasionnelle. C'est une fonction de tutelle qui doit être prise dans tout son potentiel. En effet, soit un tuteur agit avec le travail tel qu'il se présente et se réalise, et profite des occasions de celui-ci sans le transformer, pour aider les apprentissages d'un autre, soit il peut intervenir et modifier le travail et ses conditions pour le rendre formateur. Certains professionnels chargés de former sont ainsi ou non autorisés, malgré qu'ils puissent ou non se sentir autorisés et compétents pour le faire. Cela pourrait faire partie d'une formation qui les préparerait à leur rôle.

La deuxième fonction est évidemment celle d'aide, de guidage, d'explication, etc. qui recouvre un vaste ensemble d'actions possibles que nous n'aborderons pas ici. Mais, là encore, même si le déroulement du travail et l'organisation des situations ne sont pas affectés directement et explicitement, la situation pour apprendre n'est plus la même. Elle est enrichie et élargie par la présence active de celui qui forme en situation, mais surtout avec la situation. 


\section{REVUE HYBRIDE DE L'ÉDUCATION}

\section{Références}

Bruner, J. (1983). Savoir-faire, savoir dire. PUF.

Bruner, J. (1988). Comment les enfants apprennent à parler. Retz.

Bruner, J. (2000). Culture et modes de pensée. Retz.

Dewey, J., Meuret, D., Zask, J. et Deledalle, G. (2011). Démocratie et Éducation suivi de Expérience et Éducation. Armand-Colin.

Jullien, F. (2017, août). Philosophie de François Jullien. Des concepts proposés à l'art. Artpress2, (46), 113.

Le Boterf, G. (2017). De l'ingénierie de la formation à l'ingénierie de professionnalisation. Dans P. Carré et P. Caspar (dir.), Traité des sciences et des techniques de la formation (4e éd., p. 407-424). Dunod.

Macler, S. (2017). Quelques effets non prémédités de l'analyse du travail sur les compétences de tutorat du chef de très petite entreprise [thèse de doctorat inédit]. Université de Bourgogne Franche-Comté.

Mayen, P. (2013). Les limites de l'expérience. Dans J.-M. Barbier et J. Thiévenaz (dir.), Le travail de l'expérience (p.293-314). L'Harmattan.

Mayen, P. (2017). L'ignorance et la dépendance, facteurs d'apprentissage dans les interactions de tutelle au travail. Recherche et formation, 84(1), 9-23. DOI : 10.4000/rechercheformation.2754

Mayen, P. et Gagneur, C.-A. (2017). Le potentiel d'apprentissage des situations : une perspective pour la conception de formations en situations de travail. Recherches en éducation, 28, 70-83.

Métral, J.-F. (2017). Penser la didactique pour la formation professionnelle. Recherches en éducation, 3-12. https://hal-agrosup-dijon.archivesouvertes.fr/hal-02102358/document

Pastré, P. (1999). La conceptualisation dans l'action : bilan et nouvelles perspectives. Éducation Permanente, 139(2), 13-35. https://halcnam.archives-ouvertes.fr/hal-02279882/document

Pham Quang, L. et Rémery, V. (2016). Interactions tutorales et apprentissages en situation de travail, Recherche et Formation, 83(2), 9-24.

Vygotski, L.S. (1997). Pensée et langage (3e éd.). La Dispute. 